\title{
ON A THEOREM OF HAUPT AND WIRTINGER CONCERNING THE PERIODS OF A DIFFERENTIAL OF THE FIRST KIND, AND A RELATED TOPOLOGICAL THEOREM
}

\author{
MURRAY GERSTENHABER ${ }^{1}$
}

On a closed Riemann surface $R_{\boldsymbol{g}}$ of genus $g$ there exist $g$ linearly independent differentials of the first kind, $w_{1}, \cdots, w_{g}$, and their integrals around $2 g$ canonical cycles or retrosections, $a_{1}, \cdots, a_{0}$, $b_{1}, \cdots, b_{0}$, are usually put together to form a $g \times 2 g$ matrix $\left(\alpha_{i j} ; \beta_{i j}\right)$ $=(A ; B), i, j=1, \cdots, g$, where $\alpha_{i j}$ is the integral of $w_{i}$ over $a_{j}$. Riemann showed that the entries in this matrix were far from arbitrary. In fact, both of the square matrices $A$ and $B$ are nonsingular, making it possible to choose a new basis for the set of differentials of the first kind in such a way that $A$ is replaced by the $g \times g$ identity matrix and $B$ is replaced by $A^{-1} B$. The whole $g \times 2 g$ matrix is then of the form $(I, Z)$ where $Z=A^{-1} B$ is a square matrix with complex entries. If each element of $Z$ is written as the sum of its real and imaginary parts, then $Z$ itself may be written as a sum of two matrices, $Z=X+i Y$, where $X$ and $Y$ have real entries. Then the Riemann relations between the entries in $(A ; B)$ are equivalent to the statement that $Z$ is now symmetric (from which it follows that both $X$ and $Y$ are symmetric) and that $Y$ is in fact positive definite.

An arbitrary $g \times 2 g$ matrix $(A ; B)$ where $A$ is nonsingular and $A^{-1} B$ is symmetric and has positive definite imaginary part is often called (after Scorza) a Riemann matrix, but it is not true that every Riemann matrix, in this sense, arises as the Riemann matrix associated with a Riemann surface. Let $(A ; B)$ be an arbitrary Riemann matrix. ${ }^{2}$ Since $Z=A^{-1} B$ is symmetric, it has $g(g+1) / 2$ independent complex entries, which may be taken as the coordinates of a point in complex $g(g+1) / 2$-space, $C^{o(0+1) / 2}$. The set of points arising thus from the totality of all Riemann matrices of genus $g$ is an open set, being restricted only by the condition that the imaginary part of $Z$ be positive definite, and therefore has the same dimension as the space. But it is known that if the set of all Riemann surfaces of genus $g$ is parametrized in any reasonable fashion, then for $g=1$, one complex param-

Received by the editors October 28, 1952.

${ }^{1}$ Frank B. Jewett Fellow.

2 The reader is warned that more general matrices are in fact usually called Riemann matrices (cf. Zariski, Algebraic surfaces, p. 136 or Albert, Structure of algebras, p. 188). These are not relevant to the present discussion; the sense of "Riemann matrix" is here, therefore, restricted. 
eter is needed, and for higher $g, 3 g-3$ complex parameters are required. Therefore, for $g \geqq 4$ the dimension of the set of all Riemann matrices is too great for every Riemann matrix to have come from a Riemann surface. For $g=4$ the complex dimension is too large by one, and one would suppose that there exists a complex analytic function defined in $C^{10}$ which vanishes at every point whose coordinates are entries in a Riemann matrix which does come from a Riemann surface of genus 4 and no others. This matter was studied by Poincaré, and Schottky [3] later gave the necessary relation in terms of $\vartheta$ functions.

For $g=1,2$, and 3, the sets of Riemann matrices and Riemann surfaces coincide in dimension, and so for these genera one can not hope to find any other functional relation between the elements of a Riemann matrix coming from a Riemann surface than those found by Riemann. In fact, it is shown by elementary considerations that every Riemann matrix of genus one does come from a Riemann surface of genus one. But for genus two and genus three, despite the coincidence in dimension, this is lamentably not the case.

Although the necessary and sufficient conditions that a $g \times 2 g$ matrix be a Riemann matrix associated with a Riemann surface are still wrapped in mystery, the necessary and sufficient conditions that a set of $2 g$ complex numbers $\alpha_{1}, \cdots, \alpha_{0}, \beta_{1}, \cdots, \beta_{0}$ be the periods of a differential of the first kind, $w$, are known and are due to Otto Haupt [1]. The Riemann relations on the Riemann matrix of a Riemann surface imply certain conditions on the $\alpha$ and $\beta$. Haupt proved that, with one exceptional case, every set of $2 g$ complex numbers satisfying these conditions can be made to serve as periods of a differential of the first kind on some Riemann surface. To explain the exception, it must be observed that if the set of retrosections $a_{1}, \cdots, a_{0}, b_{1}, \cdots, b_{0}$ is replaced by another, then the periods of a differential of the first kind will also be changed, and in a manner determined by the change in retrosections.

The new set of periods may be considered equivalent to the old, for if either can be realized as the periods of some differential, then so can the other. The exception is this: For $g>1$ no set of presumptive periods of which only two are not zero, or which is equivalent to a set in which only two are not zero, can be realized as the periods of a differential of the first kind.

Haupt proved that it is sufficient to exclude this exceptional case, and showed that it was indeed necessary to do so by giving a proof due to Wirtinger of the following theorem: If on a Riemann surface there exists a differential of the first kind whose period system con- 
tains only two nonzero periods, or whose period system is equivalent (under change of retrosection) to one containing only two nonzero periods, then the genus of the surface is necessarily one. The proof depends on the use of the Weierstrass $\sigma$-function and the Weierstrass gap theorem.

Now

$$
\left(\begin{array}{llll}
1 & 0 & i & 0 \\
0 & 1 & 0 & i
\end{array}\right)
$$

is a perfectly good Riemann matrix of genus two. But by the theorem of Haupt and Wirtinger, 1, 0, i, 0 can not be the periods of any differential of the first kind, and therefore it is not the Riemann matrix of any Riemann surface. The case for genus three is similar. The fact that certain Riemann matrices of genus two and three do not come from Riemann surfaces may then be considered as a consequence of the Haupt-Wirtinger theorem. Since this theorem has, therefore, at least one important corollary, it may be of interest to examine some proofs longer but less sophisticated than that of Wirtinger.

Suppose then that a Riemann surface $S_{0}$ of genus $g$ with retrosections $a_{1}, \cdots, a_{g}, b_{1}, \cdots, b_{g}$ is given, and on it a differential of the first kind $w$ whose periods over these retrosections are $\alpha_{1}, \cdots, \alpha_{0}$, $\beta_{1}, \cdots, \beta_{0}$ respectively. Of these periods, suppose only two are nonzero. Since it can not be the case that all the $\alpha$ or all the $\beta$ are zero, one of these nonzero periods must be an $\alpha$ and one a $\beta$. We may therefore assume that $\alpha_{1}$ is not zero, and by multiplying $w$ by a constant factor we may assume that $\alpha_{1}=1$. It must then be the case that $\beta_{1}$ is not zero and has, in fact, positive imaginary part, which follows from the fact that if $(I, Z)$ is a Riemann matrix then the imaginary part of $Z$ is positive definite.

If $q$ is a fixed point on $S_{o}$, then the mapping $p \rightarrow z(p)=\int_{a}^{p} w$ maps $S_{o}$ into the complex plane, but since the integral depends on the path of integration it is not single-valued and the image of a point $p$ is therefore not unique. In fact, if $z_{0}(p)$ is any image of $p$, then $z_{0}(p)$ $+m \alpha_{1}+n \beta_{1}$ is also an image of $p$. As $m$ and $n$ run through all the integers, these will run through all the images of $p$ since $w$ has only the two periods $\alpha_{1}$ and $\beta_{1}$. If the points $z$ and $z+m \alpha_{1}+n \beta_{1}$ of the complex plane are considered equivalent and identified, the resulting Riemann surface is a torus $S_{1}$. There is a natural mapping $h$ of the complex plane $C$ onto the torus $S_{1}$ which sends every point of the complex plane to its equivalence class; $C$ is the universal covering surface of $S_{1}$ and $h$ the covering map. The combined mapping $p \rightarrow z(p)$ $\rightarrow h(z(p))$ is now a single-valued mapping of $S_{0}$ into the torus $S_{1}$ and 
will be denoted by $f . f$ is analytic in terms of the local coordinates on $S_{o}$ and $S_{1}$. It does in fact map $S_{\rho}$ onto $S_{1}$ and is a local homeomorphism except at the zeros $p_{1}, \cdots, p_{r}$ of $w$. Were it not for the points $p_{1}, \cdots, p_{r}$, which are branch points of $f$, the mapping would be a covering mapping of $S_{0}$ onto $S_{1}$. Since the integral of $w$ around any of the retrosections $a_{2}, \cdots, a_{0}, b_{2}, \cdots, b_{0}$ is zero, the images of these cycles under the mapping $p \rightarrow z(p)$ of $S_{0}$ into the universal covering of $S_{1}$ are closed curves. Therefore $f\left(a_{2}\right), \cdots, f\left(a_{o}\right), f\left(b_{2}\right), \cdots, f\left(b_{o}\right)$ are all homotopic to zero.

There has been constructed, then, an analytic mapping $f: S_{0} \rightarrow S_{1}$ which, except for a finite number of points, is a covering mapping, and under which the images of $g-1$ pairs of retrosections of $S_{\boldsymbol{\sigma}}$ are homotopic to zero. The Haupt-Wirtinger theorem is contained in the statement that no such mapping can exist. This latter statement does not depend on the fact that $S_{o}$ and $S_{1}$ are Riemann surfaces and $f$ an analytic map, but we shall give first a proof that uses these properties and then one which does not.

Since $S_{1}$ is a torus, there is defined on it a differential $v$ of the first kind which is mapped by a map associated with $f$ onto a differential of the first kind on $S_{0}$. Since $f\left(a_{2}\right), \cdots, f\left(b_{g}\right)$ are homotopic to zero, the latter differential can have nonzero periods only over the cycles $a_{1}$ and $b_{1}$, and therefore can differ from $w$ only by a constant multiplier. We may therefore assume it is $w$ and then the integral of $w$ over a path in $S_{o}$ is equal to the integral of $v$ over its image. If the exterior products $(i / 2) w \wedge \bar{w}$ and $(i / 2) v \wedge \bar{v}$ are taken as differentials of area on $S_{o}$ and $S_{1}$ respectively, then $(i / 2) \int w \wedge \bar{w}$ (which is just the Dirichlet integral of $w$ ) extended over all $S_{g}$ is the area of the domain onto which $f$ maps $S_{o}$, where the latter area is measured with the differential of area $(i / 2) v \wedge \vec{v}$ and domains of $S_{1}$ which are multiply covered are counted with their multiplicities. $f$ is then an isometry.

Since $f\left(a_{1}\right)$ and $f\left(b_{1}\right)$ constitute a set of retrosections of $S_{1}$, the total area of $S_{1}$ is $R\left(\alpha_{1}\right) I\left(\beta_{1}\right)-R\left(\beta_{1}\right) I\left(\alpha_{1}\right)$, where $R(\alpha)$ is the real part of $\alpha$ and $I(\alpha)$ is its imaginary part. The total area of $S_{0}$ is determined by the periods $\alpha_{1}, \cdots, \alpha_{0}, \beta_{1}, \cdots, \beta_{0}$ of $w$ and is $\sum_{i}\left[R\left(\alpha_{i}\right) I\left(\beta_{i}\right)\right.$ $\left.-R\left(\beta_{i}\right) I\left(\alpha_{i}\right)\right]$. Since $\alpha_{2}, \cdots, \alpha_{g}, \beta_{2}, \cdots, \beta_{g}$ are by assumption zero, the sum reduces to its first term, which is just the area of $S_{1}$. It follows that $S_{1}$ is not multiply. covered at all and that $f$ is therefore one-to-one. $f$ is therefore a homeomorphism, and the genus $g$ must have been one from the start. This ends the analytic proof. It should be observed that since $f$, after excepting a finite number of points, is a covering map, every two subdomains of $S_{1}$ are covered by $S_{o}$ with equal multiplicities. The degree of $f$ is therefore the ratio of the area 
of $S_{o}$ to that of $S_{1}$. Under the stringent conditions imposed on $f$, this was shown to be one.

The matter is now reduced to its topological essence. Suppose a mapping $f: S_{g} \rightarrow S_{1}$ of a surface of genus $g$ into a torus is given which, except for a finite number of branch points, is a covering mapping. The mapping must be onto for elementary topological reasons. If the degree of the mapping is one then it must be a homeomorphism and $g$ must be one. Let $a_{1}, \cdots, a_{g}, b_{1}, \cdots, b_{0}$ be a set of retrosections of $S_{1}$. Suppose $f\left(a_{2}\right), \cdots, f\left(a_{o}\right), f\left(b_{2}\right), \cdots, f\left(b_{o}\right)$ are all homotopic to zero. Then it will be shown that a new torus $S_{1}^{*}$ and a new mapping $f^{*}: S_{g} \rightarrow S_{1}^{*}$ can be constructed such that $f^{*}$ is also, except for a finite number of branch points, a covering map, but has the further property that its degree is one. $f^{*}$ must be then a homeomorphism and $g$ must be one.

If $c_{1}$ and $c_{2}$ are any two cycles of $S_{1}$, their intersection number will be denoted by $\left\langle c_{1}, c_{2}\right\rangle$. Then it is the case, as was shown by Hopf [2], that the degree of any continuous mapping $f: S_{0} \rightarrow S_{1}$ is $\sum_{i}\left\langle f\left(a_{i}\right), f\left(b_{i}\right)\right\rangle$. Since, after excepting a finite number of branch points, our $f$ is a covering mapping, its degree is not zero. But $f\left(a_{2}\right), \cdots, f\left(a_{g}\right), f\left(b_{2}\right), \cdots, f\left(b_{g}\right)$ are all homotopic to zero and so $\left\langle f\left(a_{1}\right), f\left(b_{1}\right)\right\rangle$ is not zero. It is then possible to construct a torus $S_{1}{ }^{*}$ with retrosections $a^{*}$ and $b^{*}$ and a covering mapping (a genuine one, without branch points) $h: S_{1}^{*} \rightarrow S_{1}$ such that $h\left(a^{*}\right)$ is homotopic to $f\left(a_{1}\right)$ and $h\left(b^{*}\right)$ is homotopic to $f\left(b_{1}\right)$. Let $x_{g}, x_{1}$, and $x_{1}^{*}$ be base points for the fundamental groups of $S_{g}, S_{1}$, and $S_{1}^{*}$ such that $f\left(x_{0}\right)=x_{1}$ $=h\left(x_{1}^{*}\right) . S_{g}$ may then be mapped into $S_{1}^{*}$ as follows: If $p$ is any point of $S_{g}$, let it be joined to $x_{0}$ by a curve $c . f(c)$ is a curve in $S_{1}$ with end point $x_{1}$. Over it in $S_{1}^{*}$ lies a curve with end point $x_{1}^{*}$; the other end point is to be the image of $p$. The mapping so defined is $f_{1}^{*}$. Except for the branch points of $f$ it is a covering mapping, and it is well-defined. To prove the latter point one must show that the image of a closed curve in $S_{g}$ is a closed curve in $S_{1}^{*}$. But this is true if it is true for the generators of the fundamental group of $S_{g}$; it is true for $a_{2}, \cdots, a_{g}, b_{2}, \cdots, b_{g}$ since their images under $f$ are homotopic to zero and it is true for $a_{1}$ and $b_{1}$ since $h\left(a^{*}\right)$ is homotopic to $f\left(a_{1}\right)$ and $h\left(b^{*}\right)$ is homotopic to $f\left(b_{1}\right)$. It follows, in fact, that $f^{*}\left(a_{2}\right), \cdots, f^{*}\left(a_{0}\right)$, $f^{*}\left(b_{2}\right), \cdots, f^{*}\left(b_{g}\right)$ are closed curves homotopic to zero and that $f^{*}\left(a_{1}\right)$ is homotopic to $a^{*}$ and $f^{*}\left(b_{1}\right)$ to $b^{*}$. But by Hopf's formula, $f^{*}$ then has degree one; it is a homeomorphism.

This ends, for the present paper, the discussion of the HauptWirtinger theorem, but there remain a few remarks germane to the underlying topological question. Every continuous mapping $f: S_{\sigma} \rightarrow S_{1}$ 
induces a homomorphism of the homology group of $S_{0}$ into that of $S_{1}$. Hopf showed (in the same paper [2]), that two mappings are homotopic if and only if they induce the same homomorphism, and that for every homomorphism there was a mapping inducing it. It has been shown here that if the homomorphism annihilates $g-1$ pairs of a set of retrosections of $S_{g}$, then the mapping inducing it can not have the very special property of being except for branch points a covering mapping, nor can it be homotopic to such a mapping.

Now it is in fact the case that if the homomorphism induced by a mapping $f: S_{g} \rightarrow S_{1}$ is well-behaved to the extent that however the retrosections of $S_{g}$ may be chosen it does not annihilate any $g-1$ pairs of them, then $f$ must be homotopic to a mapping which except for a finite number of branch points is a covering mapping. This can be shown by exhibiting for every such homomorphism a mapping of this special type inducing it, but that is a matter with which I do not here intend to bore you.

\section{REFERENCES}

1. Otto Haupt, Ein Satz ïber die Abelsche Integrale 1. Gattung, Math. Zeit. vol. 6 (1920) pp. 219-237.

2. Heinz Hopf, Beiträge sur Klassifizierung der Flächenabbildungen, J. Reine Angew. Math. vol. 165 (1931) pp. 225-236.

3. Friedrich Schottky, Zur Theorie der Abelschen Functionen von vier Variabeln, J. Reine Angew. Math. vol. 102 (1888) pp. 304-352.

The Institute for Advanced Study 